\title{
Optically controlled slow and fast light in optical fibers using stimulated Brillouin scattering
}

\author{
Miguel González-Herráez, ${ }^{\text {a) }}$ Kwang-Yong Song, ${ }^{\text {b) }}$ and Luc Thévenaz \\ Nanophotonics and Metrology Laboratory, Ecole Polytechnique Federale de Lausanne, STI-NAM Section \\ 11, Lausanne CH-1015, Switzerland.
}

(Received 18 January 2005; accepted 6 July 2005; published online 18 August 2005)

\begin{abstract}
We demonstrate a method to achieve an extremely wide and flexible external control of the group velocity of signals as they propagate along an optical fiber. This control is achieved by means of the gain and loss mechanisms of stimulated Brillouin scattering in the fiber itself. Our experiments show that group velocities below $71000 \mathrm{~km} / \mathrm{s}$ on one hand, well exceeding the speed of light in vacuum on the other hand and even negative group velocities can readily be obtained with a simple benchtop experimental setup. We believe that the fact that slow and fast light can be achieved in a standard single-mode fiber, in normal environmental conditions and using off-the-shelf instrumentation, is very promising for a future use in real applications. (c) 2005 American Institute of Physics.

[DOI: $10.1063 / 1.2033147]$
\end{abstract}

The active control of the speed of a light signal in an optical fiber is attracting much attention for the development of fast-access memories and optically controlled delay lines compatible with optical computing and fiber-optic communication systems. To date, there is no known method of realizing such an optically controlled delay line in optical fibers, although they are believed necessary for the development of the future all-optical packet routers. Successful experiments to widely control the light group velocity have been widely reported these past few years, ${ }^{1}$ showing the possibility to slow the speed of light up to nearly stopping $\mathrm{it}^{2,3}$ or to achieve group velocity exceeding the vacuum light velocity $c .{ }^{4,5}$ Strong negative group velocities have also been demonstrated. ${ }^{6}$ But all these experiments use special media like cold atomic gases ${ }^{4-6}$ or electronic transitions in crystalline solids ${ }^{7}$ working at well defined wavelengths. Previous works have demonstrated the possibility of achieving superluminal group velocities in optical fibers, ${ }^{8}$ but with no optical control. This letter reports the first demonstration of a wide optical control of the signal velocity in an optical fiber, realized with the recently suggested approach of using the narrow band gain or loss generated by a nonlinear optical interaction, the stimulated Brillouin scattering. 9 The high flexibility of this interaction makes this active control possible in any type of fiber and at any wavelength, in particular in the low loss window of optical fibers.

Stimulated Brillouin scattering (SBS) is usually described as the interaction of two counterpropagating waves, a strong pump wave, and a weak probe wave. ${ }^{10}$ If particular phase matching conditions are met (namely $f_{\text {Pump }}=f_{\text {probe }}$ $+\nu_{B}, \nu_{B}$ being the Brillouin shift), an acoustic wave is generated. This acoustic wave scatters photons from the pump to the probe wave, stimulating the process. From a practical point of view, the process of SBS can be viewed as a narrowband amplification process, in which a continuous-wave

\footnotetext{
a) On leave from: Dept. of Electronics, University of Alcalá, Madrid 28805, Spain; electronic mail: miguelg@depeca.uah.es

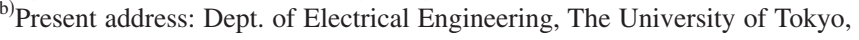
Japan.
}

pump produces a narrowband $(30-50 \mathrm{MHz})$ gain in a spectral region around $f_{\mathrm{Pump}}-\nu_{B}$.

Assuming $f_{\text {Pump }}=f_{\text {probe }}+\nu_{B}$ and no pump depletion, the spatial evolution of the electric field amplitudes of pump $\left(A_{p}\right)$ and probe $\left(A_{s}\right)$ waves under SBS is described by the following coupled equations:

$$
\begin{aligned}
& \frac{d A_{p}}{d z}=-\frac{g_{B}}{2 A_{\text {eff }}} \frac{\left|A_{s}\right|^{2}}{1-2 j\left(\frac{\Delta \nu}{\Delta \nu_{B}}\right)} A_{p}-\frac{\alpha}{2} A_{p}, \\
& \frac{d A_{s}}{d z}=\frac{g_{B}}{2 A_{\text {eff }}} \frac{\left|A_{p}\right|^{2}}{1+2 j\left(\frac{\Delta \nu}{\Delta \nu_{B}}\right)} A_{s}+\frac{\alpha}{2} A_{s},
\end{aligned}
$$

where $g_{B}$ is the Brillouin gain coefficient, $A_{\text {eff }}$ is the mode effective area, $\Delta \nu$ is the frequency deviation from $\nu_{B}, \Delta \nu_{B}$ is the gain bandwidth, and $\alpha$ is the linear attenuation coefficient. The usual treatment of these equations in the literature has basically concentrated on the intensity increase of the probe wavelength, which can be described with the exponential law $I_{s}(L)=I_{S}(0) \exp \left(g_{B} I_{P} L\right)$. However, while the real parts of the equations are related with gain in the probe wave or loss in the pump wave, the imaginary parts are responsible for additional phase shifts undergone by the two waves. ${ }^{11}$ More specifically, through the SBS process the pump wave induces a propagation constant change in the probe wave given by

$$
\Delta \beta=\operatorname{Im}\left[\frac{g_{B}}{2} \frac{I_{P}}{1+2 j\left(\frac{\Delta \nu}{\Delta \nu_{B}}\right)}\right],
$$

where $I_{P}$ is the pump power intensity in the fiber. This change in the propagation constant has a strong frequency dependence, as shown in Fig. 1. If we consider a pulse propagating at the probe wavelength, its velocity will be related to the group velocity $v_{g}=(d \beta / d \omega)^{-1}$. Thus, a sudden change in the propagation constant with frequency produces a strong change in the group velocity, which in turn introduces an additional delay of the pulse at the fiber output, 


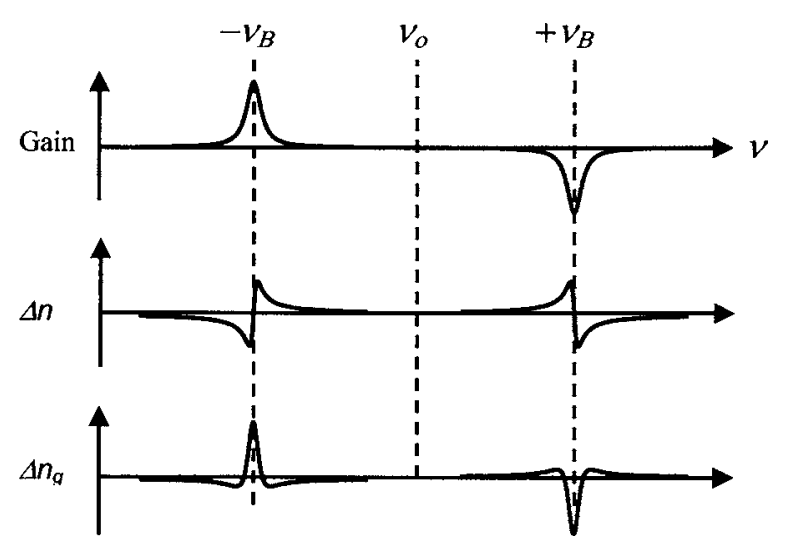

FIG. 1. Principle of signal delaying and advancement using stimulated Brillouin scattering.

$\Delta t=L / v_{g}$. If the frequency difference between pump and probe exactly matches the Brillouin shift $(\Delta \nu=0)$, the resultant optical time delay is given by $\Delta t=g_{B} I_{P} L /\left(2 \pi \Delta \nu_{B}\right)$. We can thus say that the delay varies logarithmically with the net gain (loss) experienced by the probe. A fast evaluation of these quantities in conventional single-mode fibers $\left(\Delta \nu_{B}\right.$ $\approx 35 \mathrm{MHz}$ ) leads to a surprisingly simple rule-of-thumb: 1 ns delay is introduced per decibel gain introduced in the probe.

By tuning the probe wavelength so that $f_{\text {Pump }}=f_{\text {probe }}$ $-\nu_{B}$ it is possible to observe loss at the probe wavelength. The treatment of the loss case is exactly the same to the gain case but changing the roles of pump and probe in the coupled equations, so the delay in this case is not positive but negative. This is viewed as an advancement of the pulse with respect to the conventional propagation along the fiber.

Figure 2 shows the experimental configuration, in which the pump and probe signals are generated through the modulation of the light from one laser. ${ }^{12}$ This results in an ideal stability as far as the frequency difference between pump and signal is concerned, that is essential regarding the narrow spectral width of the Brillouin gain. To properly observe the delay, a pulse probe signal is generated while the pump is a cw. A distributed feed-back (DFB) laser diode operating at $1552 \mathrm{~nm}$ was used as a light source and its output was launched into an electro-optic modulator (EOM) to create two first-order sidebands. The carrier wave was suppressed by controlling the dc bias voltage delivered into the EOM. The frequency difference between the two sidebands was set exactly to the Brillouin frequency $\nu_{B}$ of the test fiber, that is around $10.8 \mathrm{GHz}$ at this wavelength for standard fibers.

In order to measure the effect of Brillouin gain, the lower-frequency sideband was reflected by a narrow band fiber Bragg grating and optically gated to be used as a probe pulse. For this purpose another EOM was used as a fast optical gate, resulting in clean pulses with sharp rising and trailing edges. The higher-frequency sideband was used as the $\mathrm{cw}$ Brillouin pump after being amplitude controlled by a broadband erbium-doped fiber amplifier and a variable attenuator. The time delay of the probe pulse was measured for different Brillouin gains by varying the pump amplitude from zero to several tens of milliwatts. In the case of the Brillouin loss experiment, we swapped the roles of the two sidebands, so that the higher-frequency sideband was used to build the probe pulse and the lower frequency sideband was used to build the $\mathrm{cw}$ pump wave. Then, the measurement was performed in the same way by varying the power of the $\mathrm{cw}$ pump wave and monitoring the amplitude and the time delay of the probe pulse.

We first observed the delaying effect in a $11.8 \mathrm{~km}$ standard single mode fiber using a probe pulse with a full width at half maximum of $100 \mathrm{~ns}$. A maximum delay (advancement) of $30 \mathrm{~ns}(-8.4 \mathrm{~ns})$ was obtained when the Brillouin gain (loss) was $30 \mathrm{~dB}(-12 \mathrm{~dB})$. The delay varies logarithmically with the net gain with a slope of $1.07 \mathrm{~ns} / \mathrm{dB}$, in good agreement with the theoretical prediction, and the corresponding group index changes were in the order of $10^{-4}$.

Since the delay (advancement) only depends on the overall gain (loss) experienced along the full fiber length for a given type of fiber, the group index change can be drastically increased by realizing the same gain (loss) over a shorter fiber using a higher pump power. In other words, the index change will scale in the inverse proportion of the fiber length for a fixed gain (loss) to maintain the same delay. Actually the group index change will vary from the $10^{-3}$ range for kilometer-long fibers to the unity range for meterlong fibers. In this latter case it is thus possible to conceive a system with a group index smaller than 1 , hence, faster than the vacuum light velocity, or even negative, with the pulse maximum emerging out of the fiber before it actually enters the fiber.

We could experimentally verify that this extreme situation can be actually realized. The same experiment was carried out in a sample of $2 \mathrm{~m}$ of standard single mode fiber. The main issue was to raise the pump power to the $10 \mathrm{~W}$ range to obtain a $30 \mathrm{~dB}$ gain using stimulated Brillouin scattering over a short fiber. This could be achieved by forming

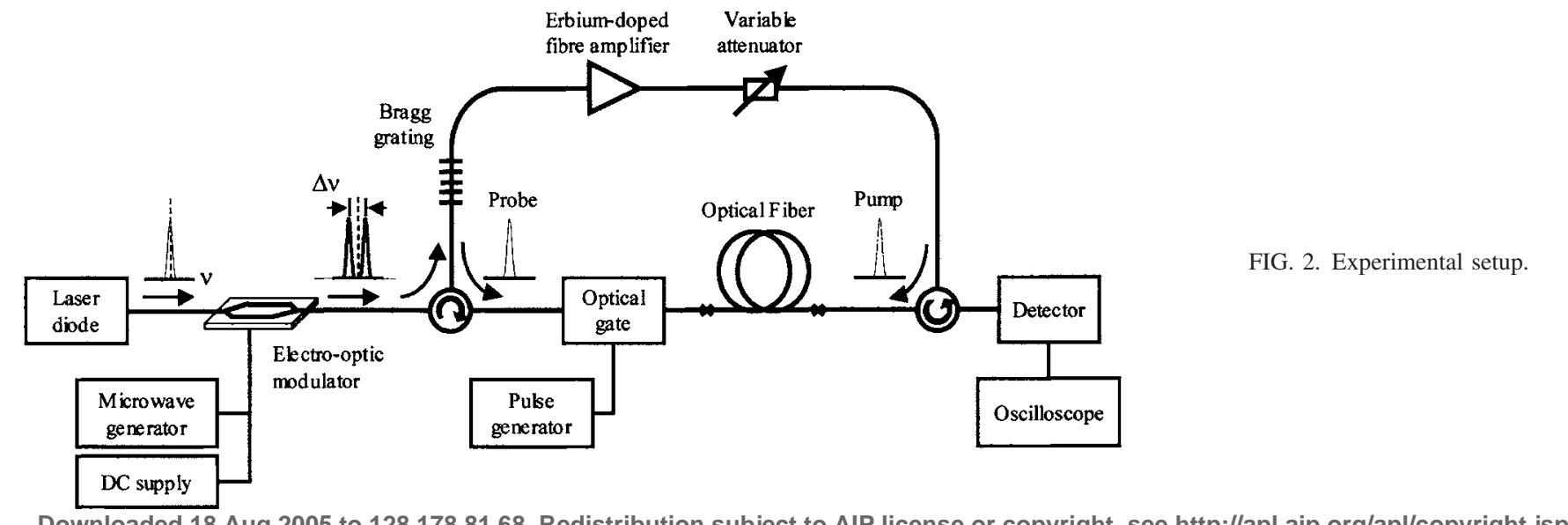




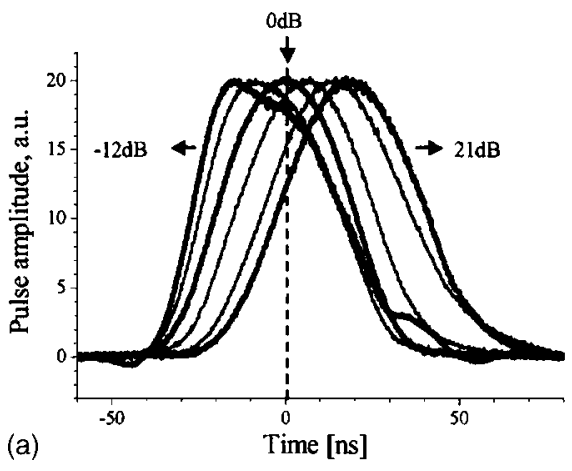

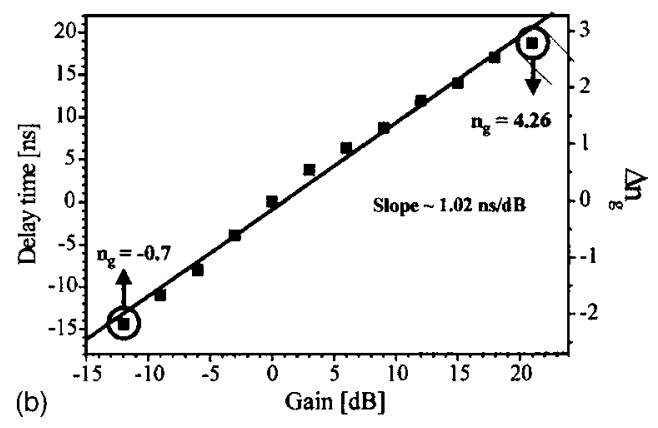

FIG. 3. (a) Pulse wave form at the fiber output. (b) Pulse delay and group index modification achieved within the fiber. a pulse train with the pump wave, so that the full gain is available in the erbium-doped fiber amplifier while the average output power is kept below the saturation power. For this purpose, an additional electro-optic modulator was used to gate the pump pulse. This gating was synchronized with the probe pulse and the pump pulse was made longer than twice the propagation time in the fiber sample, so that the signal pulse sees a constant pump power while propagating throughout the entire fiber sample.

Figure 3(a) shows time wave forms of pulses experiencing different gains and losses through stimulated Brillouin scattering in the short fiber. The observed delays are fully comparable in this $2 \mathrm{~m}$ sample to those obtained along several kilometers of fiber. Figure 3(b) shows the pulse peak position as a function of the gain (loss) experienced by the signal and the equivalent group index change. As it can be seen, this index could be increased continuously from 1.46 in normal conditions $\left(v_{g} \sim 205000 \mathrm{~km} / \mathrm{s}\right)$ to 4.26 under high Brillouin gain $\left(v_{g} \sim 70500 \mathrm{~km} / \mathrm{s}\right)$, and lowered to zero (infinite group velocity) and then to $-0.7\left(v_{g} \sim\right.$ $-428000 \mathrm{~km} / \mathrm{s}$ ) under high Brillouin loss, a negative group index meaning that the pulse peak exits the fiber before it enters. This means that the delay could be tuned from -14.4 to $+18.6 \mathrm{~ns}$ in only $2 \mathrm{~m}$ of fiber. In terms of length, this means that the fiber effective length can be continuously changed from -3 to $3.8 \mathrm{~m}$.

It must be pointed out that a group velocity faster than the vacuum light velocity $c$ or even negative does not break the famous principles resulting from causality and relativity. The fact that the spectral transition is narrowband prevents all frequency components from experiencing the same group velocity and amplitude response, so that the information cannot finally propagate at a speed faster than $c .^{5}$ This leads to a severe distortion of the pulse in the case of fast light (starting and ending points at normal light velocity in the medium and nonattenuated, peak point at modified group velocity and attenuated), resulting in a steeper leading edge and a longer trailing edge. This can be clearly observed in Fig. 3(a) in the case of strong pulse advancement. In the case of pulse delaying, the peak of the pulse is substantially amplified with respect to the leading and trailing edges, and the symmetry of the pulse appears better preserved.
To summarize, we have demonstrated a wide control of the group velocity of light signals in optical fibers using stimulated Brillouin scattering. With this effect we have achieved nearly all results obtained using atomic transitions, from delays widely exceeding the optical pulse duration ${ }^{13}$ to superluminal propagation and even negative group velocity. This experiment can be realized on a tabletop in normal environmental conditions, so that it could be the platform for the development of a wide range of applications. From the point of view of pulse control in optical communications, some mechanism has to be put forward to broaden the bandwidth of the interaction. This can be achieved by a spectral broadening $\Delta \nu_{P}$ of the pump, so that the effective Brillouin gain width is increased to $\Delta \nu_{\text {Beff }}=\Delta \nu_{B}+\Delta \nu_{P}$. But maintaining the same delay $\Delta t=g_{B} I_{P} L /\left(2 \pi \Delta \nu_{\text {Beff }}\right)$ requires a proportional increase of the pump intensity $I_{P}$ or the fiber effective length $L$.

M.G.-H. acknowledges support from the University of Alcalá through project UAH PI2005/076.

${ }^{1}$ R. W. Boyd and D. J. Gauthier, in Progress in Optics, edited by E. Wolf, Vol. 43, Chap. 6, p. 497.

${ }^{2}$ L. V. Hau, S. E. Harris, Z. Dutton, and C. H. Behroozi, Nature (London) 397, 594 (1999).

${ }^{3}$ C. Liu, Z. Dutton, C. H. Behroozi, and L. V. Hau, Nature (London) 409, 490 (2001).

${ }^{4}$ L. J. Wang, A. Kuzmich, and A. Dogariu, Nature (London) 406, 277 (2000).

${ }^{5}$ M. D. Stenner, D. J. Gauthier, and M. A. Neifeld, Nature (London) 425, 695 (2003).

${ }^{6}$ A. M. Akulshin, S. Barreiro, and A. Lezema, Phys. Rev. Lett. 83, 4277 (1999).

${ }^{7}$ M. S. Bigelow, N. N. Lepeshkin, and R. W. Boyd, Science 301, 200 (2003).

${ }^{8}$ N. Brunner, V. Scarani, M. Wegmuller, M. Legre, and N. Gisin, Phys. Rev. Lett. 93, 203902 (2004).

${ }^{9}$ D. J. Gauthier, "Physics and Applications of 'Slow' Light," 2nd Annual Summer School, Fitzpatrick Center for Photonics and Communication Systems, Duke University, Durham, NC, 27 July 2004.

${ }^{10}$ G. P. Agrawal, Nonlinear Fiber Optics, 2nd ed. (Academic, San Diego, CA, 1995).

${ }^{11}$ K. Y. Song, M. Gonzalez-Herraez, and L. Thévenaz, Opt. Express 13, 82 (2005).

${ }^{12}$ M. Niklès, L. Thévenaz, and Ph. Robert, J. Lightwave Technol. 15, 1842 (1997).

${ }^{13}$ K. Y. Song, M. Gonzalez-Herraez, and L. Thévenaz, Opt. Lett. 30, 1782 (2005). 